\title{
The Value of Engineering Literature and the Complex Role of its Contributors
}

\author{
George A. Pantazopoulos
}

Submitted: 14 August 2016/Published online: 25 August 2016

(C) ASM International 2016

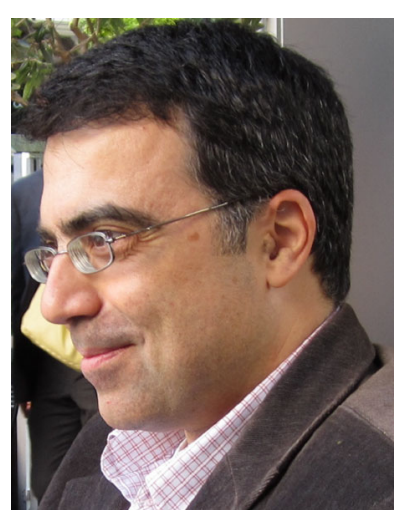

George A. Pantazopoulos

The progress of engineering disciplines is indisputably bounded with the continuous learning and production of knowledge and vice versa. According to Aristotle, knowledge is developed through experience and empirical observations which are the foundations of intellectual knowledge. Acquisition of information coming from experience interacted by intellectual cognition can be transformed to the formulation of conclusions and statements that can be considered as "elements" of knowledge. In Plato's Epistemology, knowledge is referred as the "true justified belief" and he based his knowledge system on the intellectual system of Unchanging Forms.

Scientific and Engineering literature has been tremendously extended during the last decades. Thousands of

G. A. Pantazopoulos ( $\square)$

ELKEME Hellenic Research Centre for Metals S.A., 56th km Athens - Lamia National Road, 32011 Oinofyta Viotias, Greece e-mail: gpantaz@elkeme.vionet.gr papers reflecting research works are published in Journals and announced in Conferences. Hundreds of thousands of experimental and theoretical results are exposed to the judgment and apprehension of scientific communities worldwide. In this plethora of information, the distinction and the selection of "valuable information" that could become or extract knowledge and benefit to the society is a task of unparalleled difficulty.

Beyond trends in research and chasing of projection and recognition, there is a set of fundamental and altruistic responsibilities accounted for individual members of the scientific community which share and contribute to its knowledge universe. The roles of the contributors can be expressed by the following simplified scheme (Fig. 1).

The roles are not only independent but also interacting, forming a loop of continuous improvement in engineering knowledge development through literature. Research papers and publications, which reflect and report information through theoretical and experimental evidence, constitute scripts and elements of science. The originality, usefulness, and soundness are undeniable criteria of the worthiness of publication. A brief analysis on the basic qualities contained in the above-mentioned roles (which it seems that are not quite standardized) is attempted as follows.

Reader implies to readership The scientist/engineer reader enjoys the benefits of scientific knowledge through the huge information networks, provided mainly by credible scientific literature sources, such as publishing organizations, research associations and academic institutions and libraries, research social media, internet information, etc. The reader has to trust and/or filter information channels, based on experience, judgment 


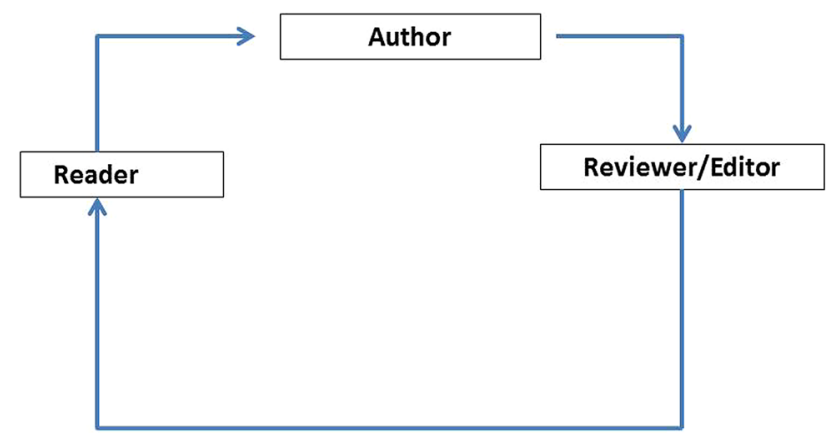

Fig. 1 Simplified schematic showing the complex role of contributing member to the "scientific knowledge society"

criteria relied on present status knowledge, reputation and prestige of information source. The validity of the information source could be cross checked and compared with other relevant research works and/or past works of the research group(s) in the specific field of the scientific subject of interest. Critical readership could drive to adoption and comprehension of ideas and theses, based on presented evidence (theory and/or experiment), which then crystallizes to knowledge. Multiple sets of metrics and quality indicators are quite helpful and reliable criteria in quantifying the impact of research in terms, e.g., of number of citations, downloads, or extent of usage of research work, aiding critical readership. Critical readership condensates the meaning of extracting the value out of the numerous published scientific papers in a certain field.

Author implies to authorship Apart from professional and academic objectives, the author's role is of critical significance and is driven by the necessity to diffuse and share scientific information which is performed by love, devotion, and enthusiasm. The romantic and altruistic perspective of offering, the almost "obsessive" desire to describe accurately and understand the natural phenomena and their causative mechanisms makes this action to occur "thermodynamically" spontaneously. Cognitive and logical models, the use of accurate and special technical/scientific language and structure, are some basic tools to assist in the creation of "elements of Scientific/Engineering literature," which is based on the outcome of the applied research work(s). The appreciation of past knowledge and experience through referenced citations, the adoption of a standard format structure, and the use of a direct writing style imposing and addressing Aristotelian "cause-and-effect" relationships can provide strong support and justification of the arguments that presented in the manuscript and also constitute direct and compelling evidence of the theses formulated. This is very important in Failure Analysis literature where the presentation of results and findings have to sufficiently support the conclusions and interpretations, following a logical thinking path.

Reviewer/Editor implies to Review and Editorship Although this role has two branches and can be envisaged as double, it is presented as one due to its controlling nature. This role reflects also the responsibilities of editorial team members and is referred to the planning, implementation, and supervision of procedures dedicated to maintain and improve the quality standards of the scientific literature. Systematic critical review must be objective and impartial and its outcome must be justifiable and delivered within a relative short time frame. Disregards the final recommendation and queries, the review could also contain, for the sake of completeness, a quick reference on the basic aspects (e.g., strengths and weaknesses) of the research paper. In Failure Analysis literature, it is very essential to ensure the soundness and the integrity of the presented results, based on standard and well-adopted methodology, which derive to safe and solid conclusions. This role (especially the Editorial one) has also additional aspects, concerning the motivation and encouraging of readership and broadening the scope of the research to the specific field in to new areas, addressing problems and exploring solutions according to, e.g., to society, market, and industrial demands.

The rapid growth of information technology and internet and, on the other hand, the development of world class publishers with a long tradition and history in the scientific literature provide the necessary "technical tools" to search, quantify, and elaborate statistics of research through indices and metrics, assisting also in increase of quality and productivity. All the above Scientific/Engineering literature "shareholders" demonstrating their complex and interacting nature of roles together also with professional associations and social, research networks, and electronic media actively work and widespread the vibrant message toward the transformation of the information society to the knowledge community. 